\title{
Strategic Signaling for Selling Information Goods
}

\author{
Shani Alkoby ${ }^{1}$, David Sarne ${ }^{2}$ and Igal Milchtaich ${ }^{2}$ \\ ${ }^{1}$ Ariel University, Ariel, Israel \\ ${ }^{2}$ Bar Ilan University, Ramat-Gan, Israel \\ shani.alkoby@gmail.com, david.sarne@biu.ac.il,igal.milchtaich@biu.ac.il
}

\begin{abstract}
This paper studies the benefit in using signaling by an information seller holding information that can completely disambiguate some uncertainty concerning the state of the world for the information buyer. We show that a necessary condition for having the information seller benefit from signaling in this model is having some "seed of truth" in the signaling scheme used. We then introduce two natural signaling mechanisms that adhere to this condition, one where the seller pre-commits to the signaling scheme to be used and the other where she commits to use a signaling scheme that contains a "seed of truth". Finally, we analyze the equilibrium resulting from each and show that, somehow counter-intuitively, despite the inherent differences between the two mechanisms, they are equivalent in the sense that for any equilibrium associated with the maximum revenue in one there is an equilibrium offering the seller the same revenue in the other.
\end{abstract}

\section{Introduction}

In this paper, we consider the problem of information providers that sell information, aiming to enable their prospective buyers a clearer characterization of the true state of the world or the true value of an opportunity they encounter. For example, an analyst selling a detailed report on a company she is covering, or services like Carfax that sell a used-car history report to a user interested in that car. Common to these examples is that the information offered by the seller enables the buyer to make a more informed decision, hence its value. The problem is that, unlike with tangible goods that are offered for sale, when it comes to information goods disclosing too many details about the information held by the seller may make it useless. For example, a used car buyer will definitely gain a lot from knowing a car she is interested in is a "lemon", however if Carfax discloses that this is indeed the case, the buyer will have no need in purchasing the full report as she will no longer be interested in the car.

While disclosing the information in full makes no sense for the seller, in many cases she can substantially benefit from partially disclosing it in the form of signaling. The idea is to provide the buyer with some indication as to the specific nature of the information held. For example, an analyst may tell you that her report has some "shocking findings" inside, without saying in what way. Unlike with selling the information itself, where buyers expect (or regulations force) truthful reporting, signaling is usually not regulated, can take almost any form, and often provides no guarantee of the truth. In many cases a signal sent can be completely uninformative. Still, signaling is widely used in real life by information sellers as a means for improving revenue [Dughmi et al., 2013; Levin and Milgrom, 2010]. Thus, we study the benefit in using signaling as a means for the information provider to increase her expected revenue from selling the information.

The paper is based on a standard model, with an information seller, an information buyer, and uncertainty about the state of the world that can be disambiguated by the information seller [Bergemann et al., 2017]. The revenuemaximizing price for the seller is equal to the value of perfect information for the buyer. This basic model is augmented by the option of using signaling for the purpose of increasing the seller's revenue. The paper provides a game theoretic analysis of this strategic signaling model.

The analysis makes three primary contributions. First, it identifies two necessary conditions that must hold in order for the seller to benefit from using signaling. These relate to a minimal commitment level that must be reflected in the signal sent, and to the valuation function of the buyer. The second contribution is the characterization of the equilibrium in two natural signaling mechanism variants that satisfy the above conditions. The first mechanism is more restrictive, in the sense that it requires the information seller to pre-commit to the signaling scheme she will be using, although it does not limit the signals themselves (which means that they can be completely unreliable). In the second mechanism, there is no pre-commitment, but in the signals themselves the seller needs to commit to a truthful elimination of some states of the world, thus reliably reducing the uncertainty associated with the true state of the world to some extent. Finally, the paper provides a proof that, somewhat counter-intuitively, despite the inherent differences between the two mechanisms, they are equivalent in the sense that for any equilibrium associated with the maximum revenue for the information seller in one mechanism there is an equilibrium offering the same revenue in the other. The importance of the last result is that it extends 
the applicability of signaling: if the information seller cannot provide evidence for truthful elimination of world states, she can commit to a signaling scheme instead, and vice versa.

\section{The Model}

We consider a standard model of an information seller (henceforth, "seller") and an information buyer ("buyer"). Both players are fully rational and self-interested, and their actions are driven by their respective beliefs of the state of the world. The (finite) set of possible states $A^{*}=\left(a_{1}, a_{2}, \ldots, a_{n}\right)$ and the prior probabilities $p^{*}: A^{*} \rightarrow[0,1]\left(p^{*}\left(a_{i}\right)>0\right.$ for all $\left.i, \sum_{i} p^{*}\left(a_{i}\right)=1\right)$ are known to both players, however the seller also knows the true state of the world, and can sell this information to the buyer. Since the seller only sells information and not a tangible good, her revenue is equal to the price she sets for the information if it is purchased, and zero otherwise. In addition to setting the price of the information, the seller may provide the buyer with a signal, attempting to influence the latter's purchase decision. The seller is allowed to send the signal only once, before the buyer makes her decision of whether to purchase the information. Along with the signal, the seller tells the buyer the price of providing her with the information. Finally, the buyer needs to make her decision of whether to purchase the information for the requested price.

We use $V(p)$ to denote the benefit for the buyer from knowing that the probability distribution of states is $p$. The function $V$ is assumed to be known to both the buyer and the seller. The goal of the seller is to maximize her expected revenue from selling the information and the goal of the buyer is to maximize her expected profit, taking into account both the benefit from purchasing the information and its cost.

\section{Analysis}

We use $S$ to denote the (infinite) set of possible signals for the seller. A deterministic signaling scheme is a function $\pi: A^{*} \rightarrow S$, that specifies the signal $\pi\left(a_{i}\right)=s \in S$ that will be sent when the true state of the world is $a_{i}$. A strategy for the seller is a specific signaling scheme $\pi$ and a corresponding price scheme, which specifies the price $c$ requested for disclosing the true state of the world for every signal used by $\pi$. Upon observing a signal $s$, the buyer's response should rely on the posterior probability function, denoted $p_{s}$, which is calculated as follows:

$$
p_{s}\left(a_{i}\right)= \begin{cases}\frac{p^{*}\left(a_{i}\right)}{\sum_{a \in \pi^{-1}(\{s\})} p^{*}(a)} & \text { if } \pi\left(a_{i}\right)=s \\ 0 & \text { otherwise }\end{cases}
$$

where $\pi^{-1}(\{s\})$ is the set of all states at which the signal $s$ is sent. ${ }^{1}$ In case of not purchasing the information from the seller (after receiving the signal $s$ ), the buyer's benefit is $V\left(p_{s}\right)$. If the information is purchased, then with probability $p_{s}\left(a_{i}\right)$ the true state is $a_{i}$ and the benefit is $V\left(a_{i}\right)=V(p)$, where $p$ assigns a probability of 1 to $a_{i}$ and 0 to all other

\footnotetext{
${ }^{1}$ We note that for the buyer to be able to update the probability correctly, she needs to get some sort of commitment from the seller regarding the signaling scheme the seller is about to use. This will be elaborated in Section 4.
}

states. More generally, we use $V(A)\left(A \subseteq A^{*}\right)$ to denote the expected benefit of the buyer from knowing that only states in $A$ are possible. Thus, $V(A)=V(p)$, where $p\left(a_{i}\right)=p^{*}\left(a_{i}\right) / \sum_{a \in A} p^{*}(a)$ if $a_{i} \in A$ and $p\left(a_{i}\right)=0$ otherwise. The buyer will purchase the information only if the expected improvement in her benefit due to receiving the full information is greater than the $\cos t c$, that is, if

$$
\sum_{a_{i} \in A^{*}} p_{s}\left(a_{i}\right) V\left(a_{i}\right)-V\left(p_{s}\right) \geq c
$$

The seller will therefore set the price of information, $c$, for every signal $s$ to be

$$
\max \left(\sum_{a_{i} \in A^{*}} p_{s}\left(a_{i}\right) V\left(a_{i}\right)-V\left(p_{s}\right), 0\right)
$$

where a zero price of information means that the information is being offered to the buyer for a cost of zero and as a result, the dominant strategy of the buyer, in case the value of the information is positive, is to purchase the information (i.e., beneficial information will be given away for no cost). Therefore, from now on, we will rely only on $\pi$ when referring to the seller's strategy, as the price of information derives from the signaling scheme used.

We continue with providing some necessary features of the signaling scheme and the underlying mechanism that must hold in order for the seller to benefit from signaling in equilibrium. For this purpose, we denote the seller's expected revenue when using signaling as $R_{\text {seller }}^{\text {signal }}$, and when not using signaling as $R_{\text {seller }}^{\neg \text { signal }}$.

Definition 1 A signaling scheme contains a "seed of truth" if it involves at least one signal that credibly excludes at least one state of the world.

Theorem 1 presents a necessary condition over the seller's signaling scheme that must hold in order for the seller to benefit from signaling.

Theorem $1 R_{\text {seller }}^{\text {signal }}$ can be greater than $R_{\text {seller }}^{\neg \text { signal }}$ only if the signaling scheme used contains a "seed of truth".

Sketch of Proof. If the signaling scheme does not contain a "seed of truth", then it is the seller's best interest to always provide the signal that maximizes her revenue, regardless of the true state of the world. Therefore, the signal sent will not change the probabilities the buyer associates with the possible world states (see Equation 1), hence will not change the amount she is willing to pay for the information. $\square$

Next, Theorem 2 provides a necessary condition over the benefit function $V$ that must hold in order for the seller to benefit from signaling. For this purpose we will first define the concept of a "potentially harmful information".

Definition 2 Information is potentially harmful for the buyer if there exists at least one pair of disjoint nonempty sets $A, B \subseteq A^{*}$ such that $V(A \cup B)>p^{*}(A \mid A \cup B) V(A)+$ $p^{*}(B \mid A \cup B) V(B) .{ }^{2}$ Thus, if the buyer knows that the true

${ }^{2} A \cup B$ means that only world states included in $A$ or $B$ are plausible. 
world state is in $A$ or in $B$, she would prefer not knowing which of these two possibilities obtains.

An example for a "potentially harmful information" can be the case of a country club owner who sells daily entrance tickets in a fixed price $Z$. Visitors' utility from visiting the country club, expressed in monetary terms, depends on the weather, and is given by $X$ (where $X>Z$ ) for a sunny day, $W$ (where $X>W>Z$ ) for a cloudy day, and $Y$ (where $Y<Z$ ) for a rainy day. Assume the prior probability for sunny is $p_{1}$, for cloudy is $p_{2}$, and for rainy is $1-\left(p_{1}+p_{2}\right)$. Now consider a weatherman who can publicly provide the exact weather condition for the coming weekend. If $X p_{1}+W p_{2}+Y\left(1-p_{1}-\right.$ $\left.p_{2}\right)>Z$ and $W \frac{p_{2}}{\left(1-p_{1}\right)}+Y \frac{\left(1-p_{1}-p_{2}\right)}{\left(1-p_{1}\right)}<Z$ then according to Definition 2 the information is harmful $(\mathrm{A}=\{$ cloudy, rainy $\}$, $\mathrm{B}=\{$ sunny $\}$ ). Still, if the signaling scheme used publicly rules out sunny whenever the real weather is cloudy or rainy then the owner will definitely buy the true value whenever this signal is used (as differentiating cloudy from rainy enables some profit rather than zero otherwise).

Theorem $2 R_{\text {seller }}^{\text {signal }}$ can be greater than $R_{\text {seller }}^{\neg \text { signal }}$ only if information is potentially harmful for the buyer.

Sketch of Proof. We will assume that the information is not potentially harmful for the buyer, and show that provision of free information is not beneficial for the seller. The seller's revenue when not using signaling, $R_{\text {seller }}^{\neg \text { signal }}$, equals to: $\max \left(\sum_{a} p^{*}(a) V(a)-V\left(p^{*}\right), 0\right)$. If using a signaling scheme $\pi$, the seller's expected revenue, $R_{\text {seller }}^{\text {signal }}$, equals to: $\sum_{a_{i}} p^{*}\left(a_{i}\right) \max \left(\sum_{a} p_{\pi\left(a_{i}\right)}(a) V(a)-V\left(p_{\pi\left(a_{i}\right)}\right), 0\right)$.

Since by assumption the information is not potentially harmful for the buyer then: (a) $V\left(A^{*}\right) \leq \sum_{a} p^{*}(a) V(a)$; (b) $V\left(A^{*}\right) \leq \sum_{s}\left(\sum_{a \in \pi^{-1}(\{s\})} p^{*}(a)\right) V\left(\pi^{-1}(\{s\})\right)$; and (c) $V\left(\pi^{-1}(\{s\})\right) \leq \sum_{a \in \pi^{-1}(\{s\})} p_{s}(a) V(a)$. Notice that, by definition, $V\left(A^{*}\right)=V\left(p^{*}\right)$ and $V\left(\pi^{-1}(\{s\})\right)=V\left(p_{s}\right)$. Using the last three inequalities we therefore obtain:

$$
\begin{aligned}
& R_{\text {seller }}^{\neg \text { signal }} \stackrel{(\mathrm{a})}{=} \sum_{a} p^{*}(a) V(a)-V\left(p^{*}\right) \\
& \stackrel{(\mathrm{b})}{\geq} \sum_{a} p^{*}(a) V(a)-\sum_{s} \sum_{\pi^{-1}(\{s\})} p^{*}(a) V\left(\pi^{-1}(\{s\})\right) \\
& 3=\sum_{a^{\prime}} p^{*}\left(a^{\prime}\right) \sum_{a} p_{\pi\left(a^{\prime}\right)}(a) V(a)-\sum_{a^{\prime}} p^{*}\left(a^{\prime}\right) V\left(p_{\pi\left(a^{\prime}\right)}\right) \\
& =\sum_{a^{\prime}} p^{*}\left(a^{\prime}\right)\left(\sum_{a} p_{\pi\left(a^{\prime}\right)}(a) V(a)-V\left(p_{\pi\left(a^{\prime}\right)} \stackrel{(\mathrm{c})}{=} R_{\text {seller }}^{\text {signal }}\right.\right.
\end{aligned}
$$

Thus, for any signaling scheme, and corresponding information prices, the expected revenue of the seller is at most the revenue obtained without signaling.

Based on Theorem 2 we can identify some classes of settings where the seller cannot benefit from signaling. One natural class is of settings where the buyer has to decide between performing an action or not (e.g., buy a rare diamond), where

\footnotetext{
${ }^{3}$ Law of iterated expectation.
}

the action may result in different payoffs according to the different world states (e.g., true worth of the diamond), and not performing results in a fixed fallback payoff $V_{\emptyset}$. Here for any $A \subseteq A^{*}$ we obtain $V(A) \leq \sum_{i=1}^{j} \frac{\sum_{a \in A_{i}} p^{*}(a)}{\sum_{a \in A} p^{*}(a)} V\left(A_{i}\right)$, hence information is never harmful.

There are many settings where the condition specified in Theorem 2 holds and the seller can benefit from signaling. For example, when the buyer's profit is influenced by the resulting equilibrium in a multi-agent system she is operating in, and the information revealed through signaling (as well as the information purchased from the seller), if revealed to the other agents, influences the equilibrium of the system. An example for this can be found in auctions, where the seller can provide the true value of some characteristic of the auctioned item, which influences the bidders' valuation of the item [Sarne et al., 2014]. Here, a signal sent by the seller, if becoming common knowledge, can influence the bids placed by the bidders, and accordingly influence the equilibrium expected value of the winning bid or the second-best bid (in case of a second-price auction). We use this application domain for some of the numerical examples introduced onwards.

\section{Seller's Commitment}

Between the two necessary conditions discussed above, "seed of truth" and "potentially harmful information", the former is the most important one, as it is an inherent feature of the signaling mechanism. ${ }^{4}$ In order for this requirement to hold, some enforceable commitment or means for validating the information provided by the signal should be enabled ("provable signals"). In this section we introduce, analyze and compare two natural and straightforward variants in which the "seed of truth" requirement is met. Those are the Strategy Commitment and the Truthful Elimination.

\subsection{Strategy Commitment (StC)}

In the Strategy Commitment variant, the seller has to commit in advance to the strategy (signaling scheme $\pi$ and list of prices) she is planning to use. This means that upon receiving a signal $s$, the buyer can eliminate any world state $a$ for which $\pi(a) \neq s$. Thus, the set of relevant states is $\pi^{-1}(\{s\})$ and the posterior probability distribution $p_{s}$ is given by Equation 1 . Unless $\pi$ is a constant function, the "seed of truth" condition is met. The course of the game is as follows. First, the seller publicly commits to a specific signaling scheme $\pi$ and a corresponding price list. Then, she learns the true state of the world, $a_{i}$, and sends to the buyer the signal $s=\pi\left(a_{i}\right)$ and the corresponding price according to the strategy she committed to. Finally, the buyer needs to decide whether to purchase the information about the true state of the world at the requested price. We will refer to this variant as the $\mathrm{StC}$ variant hereafter. The players' equilibrium behavior in this variant is characterized in Proposition 1.

Proposition 1 The following is a perfect Bayesian equilibrium for the StC variant: (a) a seller's strategy, $\pi$, in which the price is the maximal amount the buyer will agree to pay

\footnotetext{
${ }^{4}$ The "potentially harmful information" condition is external, thus beyond the seller's control.
} 
(as calculated in Equation 3) and there is no other strategy $\pi^{\prime}$ that provides the seller with a better expected revenue; and (b) a buyer's strategy according to which the information is purchased if and only if Equation 2 holds.

Sketch of Proof. Given a seller's strategy, the buyer's best response strategy is the one characterized in the general model analysis. The seller, being the first mover, will choose the strategy that maximizes her expected revenue.

We note that, since the seller publicly commits to a strategy, her choice induces a partition of the set of possible states of the world. The role of the signal is to point to the partition element that includes the true state; the actual message is irrelevant. Therefore, there is no loss of generality in specifying a seller's strategy in this variant as a partition of $A^{*}$ and a list of corresponding prices. This observation effectively shrinks the set of possible strategies for the seller to a finite set. ${ }^{5}$ An equilibrium can be found by going over the set of all partitions and calculating the expected revenue for the seller according to Equation 3 in order to find the partition yielding the highest expected revenue.

To illustrate the possible improvement in the seller's revenue from using signaling, consider an auction setting where an external information provider holds the information regarding the exact state of the world (e.g., the common value of the auctioned item), which is one of $\left\{a_{1}, a_{2}, a_{3}, a_{4}\right\}$, with equal probabilities. The prospective buyer of the information is an auctioneer whose profit from the elimination of different world states is given by: ${ }^{6}$ $V\left(\left\{a_{1}\right\}\right)=75.5, V\left(\left\{a_{2}\right\}\right)=24.7, V\left(\left\{a_{3}\right\}\right)=43$, $V\left(\left\{a_{4}\right\}\right)=60, V\left(\left\{a_{1}, a_{2}\right\}\right)=50.1, V\left(\left\{a_{1}, a_{3}\right\}\right)=59.3$, $V\left(\left\{a_{1}, a_{4}\right\}\right)=67.8, V\left(\left\{a_{2}, a_{3}\right\}\right)=73.9, V\left(\left\{a_{2}, a_{4}\right\}\right)=$ 42.3, $V\left(\left\{a_{3}, a_{4}\right\}\right)=51.5, V\left(\left\{a_{1}, a_{2}, a_{3}\right\}\right)=47.8$, $V\left(\left\{a_{1}, a_{2}, a_{4}\right\}\right)=53.4, V\left(\left\{a_{1}, a_{3}, a_{4}\right\}\right)=59.5$, $V\left(\left\{a_{2}, a_{3}, a_{4}\right\}\right)=42.6, V\left(\left\{a_{1}, a_{2}, a_{3}, a_{4}\right\}\right)=50.8$. Notice that $V\left(\left\{a_{2}, a_{3}\right\}\right)=73.9>33.9=\left(V\left(\left\{a_{2}\right\}\right)+\right.$ $\left.V\left(\left\{a_{3}\right\}\right)\right) / 2$, so the condition in Theorem 2 holds. In this example, there exist two equilibria, both providing a revenue of 1.39: a signaling scheme which induces the partition $\left\{\left\{a_{1}\right\},\left\{a_{2}\right\},\left\{a_{3}, a_{4}\right\}\right\}$ with the corresponding prices $(0,0,2.78)$, and one inducing a partition $\left\{\left\{a_{1}, a_{2}\right\},\left\{a_{3}, a_{4}\right\}\right\}$ with prices $(0,2.78)$. The seller's revenue from not using signaling is 0.49 , hence signaling in this case leads to a substantial increase in revenue.

\subsection{Truthful Elimination (TrE)}

In the Truthful Elimination variant, the seller can credibly eliminate outcomes using signaling, e.g., by using a device that provides a free low-resolution representation of the information (such as a web widget providing a crude description of tomorrow's temperatures). In case an outcome was explicitly eliminated by the seller's signal, this outcome cannot be the true outcome. This is equivalent to reducing the subset

\footnotetext{
${ }^{5}$ The number of possible partitions of a finite set is a bell number.

${ }^{6}$ The values represent the expected second highest bid in the auction for any information set known to the bidders, where the auctioneer is obligated to disclose the information to the bidders if purchased.
}

$A^{*}$ to $A_{s} \subseteq A^{*}$ such that it is guaranteed that only members of $A_{s}$ can be the true outcome (therefore, we will represent a signal that reduces $A^{*}$ to $A_{s}$ simply by $A_{s}$, where $A_{s}$ must include the true state of the world).

The course of the game is as follows. First, the seller learns the true state of the world and sends to the buyer the signal $s$ and the price for disclosing the true state of the world. Then, the buyer, observing the signal, needs to decide whether to purchase the information at the requested price. We will refer to this variant as the TrE variant hereafter.

The equilibrium analysis in this variant is complicated by the fact that the absence of commitment to a specific strategy means that the seller can potentially deviate from her prescribed strategy. Proposition 2 characterizes the equilibrium behavior. $^{7}$

Proposition 2 The following is a perfect Bayesian equilibrium for the TrE variant: (a) a seller's strategy, $\pi$, in which the price is set according to Equation 3 and there is no $\pi^{\prime}$ yielding a higher expected revenue as long as the buyer uses the best response strategy to $\pi$ outlined next; and (b) a buyer's strategy according to which the information is purchased if and only if the signal $s$ and the requested price $c$, are such that, for some state $a, \pi(a)=s$ and Equation 2 holds. If such an a does not exist, the buyer believes that, with probability 1, the true state is some arbitrarily selected state, $a^{\prime}$, which justifies the decision of not purchasing the information.

Sketch of Proof. The main point is that the seller will never deviate by sending a signal $s$ (and a corresponding price) such that $\nexists a \in A^{*}$ for which $\pi(a)=s$. Otherwise, the buyer, believing that the true state of the world is $a^{\prime}$ (with probability 1), will find no value in purchasing the information, leading to a zero revenue for the seller. In all other cases, the buyer is using her best-response strategy (for the same arguments used so far) and the seller's strategy is to use a signaling scheme $\pi$ and a corresponding price list that results in at least the same expected revenue as with any other $\pi^{\prime}$ and its corresponding price list. Therefore neither of them has an incentive to deviate, given the strategies of the other player.

One trivial equilibrium that always exists in the TrE variant is when the signaling is non-informative ${ }^{8}$, i.e., no new information is being disclosed by the seller (hence with no increase in the seller's expected revenue according to Theorem 1's proof). This is formally given in Proposition 3.

Proposition 3 In the TrE variant, any strategy which includes a non-informative signaling scheme is an equilibrium strategy.

Sketch of Proof. Since the buyer is using her best-response strategy as given in Proposition 2, all we need to prove is that

\footnotetext{
${ }^{7}$ We note that although in the TrE variant the buyer is not aware of which $\pi$ the seller chose, in equilibrium, the buyer's belief regarding the seller's strategy is identical to the actual strategy the seller uses and therefore the use of Equation 1 is valid.

${ }^{8} \mathrm{~A}$ non-informative signaling scheme is one where $\pi\left(a_{i}\right)=s$ $\forall a_{i} \in A^{*}$, i.e., always providing the same signal no matter what is the state of the world.
} 
the seller's best-response strategy is to keep disclosing the same signal $s$ for every world state. Deviating to a different signal, $s^{\prime}$, in any of the possible world states in $A^{*}$, would result in a zero revenue since the buyer will not purchase the information. Therefore, the seller should stick to $s$.

We note that this last property does not recur in the StC variant since in there, the change in the seller's strategy also changes the set of signals the buyer is expecting to receive hence the seller can benefit from deviating to an informative signaling scheme. Indeed in the numerical example provided at the end of the former section, the non-informative signaling was not in equilibrium.

Note that other than requiring a "seed of truth", the signals the seller may use are not constrained in any way. Adopting the representation of signals as the subset of remaining world states, as used with the StC variant (so now a signal $s$ is actually a set of still possible world states, i.e., $s=A_{s}$ ), we may distinguish between two possible equilibrium signaling schemes: (i) Partition-Content (PC), where $A_{s} \cap A_{s^{\prime}}=\emptyset$ for every pair of distinct signals $s$ and $s^{\prime}$; and (ii) Non-PartitionContent (NPC), where the signals do not satisfy this condition and therefor do not explicitly specify a partition. For example, a possible NPC signaling scheme for a setting with four possible states is $\pi\left(a_{1}\right)=\pi\left(a_{3}\right)=\left\{a_{1}, a_{2}, a_{3}, a_{4}\right\}$ and $\pi\left(a_{2}\right)=\pi\left(a_{4}\right)=\left\{a_{2}, a_{4}\right\}$.

Proposition 4 Together with the buyer's best response strategy specified in Proposition 2, every seller's strategy which uses a PC signaling scheme is an equilibrium.

Sketch of Proof. If the strategy $\pi$ uses a PC signaling scheme, then for every state $a_{i}$ any signal other than $\pi\left(a_{i}\right)$ necessarily eliminates $a_{i}$ and therefore cannot be used at that state.

Unlike for PC schemes, not every NPC scheme is an equilibrium strategy. A strategy based on an NPC signaling scheme is an equilibrium strategy only if at every state of the world one of the following conditions holds: (i) deviation is not possible, i.e., there is no other signal allowed by the scheme to which the seller can deviate without violating the rule of truthful elimination; (ii) deviation is not beneficial, i.e., a signal as above does exist, but deviating to it would lower the seller's expected revenue. We emphasize that even when both a PC scheme and an NPC scheme induce the same partition, it is possible that the second scheme is not in equilibrium one. Proposition 5 discusses the connection between NPC and PC equilibria.

Proposition 5 For every NPC equilibrium there is a PC equilibrium yielding the same expected revenue to the seller.

Sketch of Proof. For any NPC equilibrium, we can replace each signal $s$ used by $\pi$ (which is a subset of $A^{*}$ ) with a subset only including members of $A^{*}$ for which $\pi(a)=s$, and keep the original prices. The resulting signaling scheme is a PC, and since the seller is still charging the maximum price possible for each signal, it is an equilibrium strategy. Since prices are the same and the information is purchased in same world states, the seller's revenue is the same.
Propositions 2, 4 and 5 make the equilibrium analysis a simple matter. One should consider all PC signaling schemes and calculate the corresponding prices. Then it is possible to generate all NPC equilibria simply by adding additional states of the world to the different signals, as long as the "deviation is not possible" and "deviation is not beneficial" conditions are not violated.

We use the same numerical example used for the StC variant to demonstrate that signaling can be of value to the seller also in the TrE model. Here, the seller's revenue with no signaling is 0.49 , and with signaling, we find a plethora of equilibria yielding a revenue in the range 0 to 1.39 . Thus, some equilibria offer a substantial improvement.

\section{Comparison of $\mathrm{StC}$ and $\operatorname{TrE}$}

While in both variants the seller is making a commitment related to the signal sent, the TrE variant encapsulates a weaker commitment requirement, as the seller only eliminates some states rather than having to disclose her entire strategy. This difference results in an important difference in the number and nature of the equilibria between the two variants. In both variants, it is possible to have more than one equilibrium. In the $\mathrm{StC}$ variant, all equilibria yield the seller the same (i.e., maximal) expected revenue, but in the TrE variant, there are more equilibria and they do not all yield the same revenue. In the example presented above, there are two equilibria in the $\mathrm{StC}$ variant and 88 and in the TrE one. We emphasize that in the latter case there is no guarantee which of the equilibria will actually be used. The research on multiple non-dominating equilibrium strategies in game and agents theory is quite rich [Cooper, 1999; Morris and Shin, 2000] and is beyond the scope of this paper.

Next, we explore the relation between the equilibria that hold in $\mathrm{TrE}$ and $\mathrm{StC}$. In order to do so, we first provide a lemma and a definition.

Lemma 1 For each equilibrium in the TrE variant, there is a strategy for the seller and a best-response strategy for the buyer in the StC variant such that in each state of the world information is being purchased if and only if it is purchased in the $\operatorname{TrE}$ variant, and for the same price.

Sketch of Proof. Given an equilibrium in the TrE variant, consider the seller's strategy in the $\mathrm{StC}$ variant which, for each state in $A^{*}$, specifies sending the same signal and requesting the same price as in the first equilibrium. Since the seller is the first mover, the buyer will act according to her best-response strategy as defined in the $\mathrm{StC}$ variant analysis. Now since this is an equilibrium in the $\operatorname{TrE}$ variant, the seller's strategy induces a partition of $A^{*}$. Any strategy in the $\mathrm{StC}$ variant (equilibrium or not) also induces a partition. Since both strategies above induce the same partition of $A^{*}$ and the same prices are being asked, the buyer will act the same in both variants and the seller will end up with the same revenue.

Definition 3 An equilibrium in the StC variant and an equilibrium in the TrE variant are equivalent if at every state of the world where information is purchased in one equilibrium, 
it is purchased also in the other equilibrium, and for the same price.

Based on Lemma 1 and Definition 3 we can now prove Theorem 3.

Theorem 3 The equilibria in the StC variant are equivalent to those equilibria in the $\operatorname{Tr} E$ variant that yield the highest revenue to the seller.

Sketch of Proof. If this is not so, then according to Lemma 1 there is an equivalent strategy in the $\mathrm{StC}$ variant that gives the seller a higher expected revenue. The seller can deviate to that strategy, so the original solution in the $\mathrm{StC}$ variant is not an equilibrium to begin with.

One interesting and counter-intuitive insight resulting from Theorem 3 is that although the TrE variant seems more applicable from the seller's point of view (a commitment to providing a "seed of truth" in the signaling scheme is less restrictive than a commitment to a complete strategy), she may just as well use the StC scheme and benefit at least the same. In particular, the equivalence is important in cases where truthful elimination results in multiple equilibria with different revenues to the seller, as she can simply switch to the other mechanisms in order to guarantee maximum revenue.

\section{Related Work}

The model analyzed in the paper can be considered a special case of a signaling game [Cho and Sobel, 1990; SteinertThrelkeld, 2014]. The seller is the sender, the buyer is the receiver and states of the word correspond to possible sender's types. However, in our case, unlike in the general signaling game literature, the choice of signal is tightly linked with the price that can be charged for the information provided. For example, a signal that implicitly reveals the true state of the world makes the value of the information offered for sale zero. In addition, a common assumption in the general signaling games literature is that the set of signals available to the sender is finite and known to the receiver. By contrast, the seller in our model is not limited in terms of the number or nature of signals she can use. She must only adhere to a "seed of truth" requirement that can be met in different ways, as demonstrated by the two variants analyzed above.

Much effort has been dedicated to studying optimal ways for gathering information that can reduce the uncertainty characterizing a decision situations [Shen et al., 2018; SegalHalevi et al., 2019; Das and Li, 2014]. This was investigated in a number of multi-agent domains, for example, in optimal stopping problems [Wilson et al., 2011; Azoulay-Schwartz et al., 2004] where information acquisition is costly [Moscarini and Smith, 2001; Wiegmann et al., 2010]. These works were extended to study the question of information pricing in multi-agent systems [Nahum et al., 2015; Bergemann et al., 2017; Hajaj and Sarne, 2017]. Many authors have considered the question of information revelation. For example, in auctions the auctioneer is often more informed than the bidders [Akerlof, 1978; Emek et al., 2014] and therefore can use various schemes to fully or partially reveal the information she holds in order to maximize the expected first- or second-best bid [Eső and Szentes, 2007; Ganuza and Penalva, 2010; Jewitt and Li, 2012]. Still, in all these works, the goal of information revelation is to bring the system to a more beneficial collective outcome from the information provider's perspective rather than to increase the potential worth of some information offered for sale. The power of information in strategic settings has also been widely studied [Bassan et al., 1997; Gossner, 2000]. However, most of those studies consider the problem of a normal form game as opposed to the sequential nature of our setting. Those that do consider a sequential setting, focus only on some specific domains such as economic search [Alkoby et al., 2015] or organ transplants [Hajaj et al., 2015].

The most relevant to our paper are the works of Bergemann et al. 2017 and Crawford et al. 1982. The work of Bergemann et al. also considers a self interested information seller who can resolve some uncertainty an information buyer is facing. However, in their model, signals are costly and their content is determined by nature. Crawford et al. aim to maximize joint profit from the information revealed whereas we aim to maximize the buyer's profit from the information not revealed (as this is the asset to be sold later). This substantial difference accounts for the difference in the influence signaling granularity has on profits. While in their work one should aspire to the most refined partition, this is not the case in our model and consequently one needs to evaluate all possible partitions. Finally, this work generalizes our previous work, [Alkoby et al., 2017], and provides both a richer analysis and the conditions under which signaling can be beneficial to the seller.

\section{Conclusions}

The analysis provided in the paper shows that the strategic decisions of information providers extend beyond price setting. Providers should consider the use of signals as a means for improving their revenue. As shown throughout the paper, signaling can potentially increase the value consumers attach to the full information put to sale, thus increasing their willingness to pay for it. This insight is relevant both to individuals or companies operating or developing information-providing services and to market designers.

The analysis presents two necessary conditions for signaling to be beneficial for the information seller. Those are the "seed of truth" and "potentially harmful information".

The paper analyze and compare two straightforward model variants in which the "seed of truth" requirement is met. Despite several inherent differences between the two variants, the expected equilibrium revenue for the information provider in one is equal to the maximum expected equilibrium revenue in the other. This finding is relevant for mechanism design, as it enables replacing a somewhat difficult-to-implement mechanism with a simpler one without degrading the information provider's revenue.

\section{Acknowledgments}

This research was partially supported by the ISRAEL SCIENCE FOUNDATION (grant No. 1162/17) and the ISFNSFC joint research program (grant No. 2240/15). 


\section{References}

[Akerlof, 1978] George A Akerlof. The market for "lemons": Quality uncertainty and the market mechanism. In Uncertainty in economics, pages 235-251. Elsevier, 1978.

[Alkoby et al., 2015] Shani Alkoby, David Sarne, and Sanmay Das. Strategic free information disclosure for searchbased information platforms. In $A A M A S-15$, pages 635$643,2015$.

[Alkoby et al., 2017] Shani Alkoby, David Sarne, and Igal Milchtaich. Strategic signaling and free information disclosure in auctions. In $A A A I-17$, pages 319-327, 2017.

[Azoulay-Schwartz et al., 2004] Rina Azoulay-Schwartz, Sarit Kraus, and Jonathan Wilkenfeld. Exploitation vs. exploration: choosing a supplier in an environment of incomplete information. Decision support systems, 38(1):1-18, 2004.

[Bassan et al., 1997] Bruno Bassan, Marco Scarsini, and Shmuel Zamir. "I Don't Want to Know!'” Can it be Rational? Hebrew University of Jerusalem, Center for Rationality and Interactive, 1997.

[Bergemann et al., 2017] Dirk Bergemann, Alessandro Bonatti, and Alex Smolin. The design and price of information. Technical report, Cowles foundation discussion paper no. 2049, 2017.

[Cho and Sobel, 1990] In-Koo Cho and Joel Sobel. Strategic stability and uniqueness in signaling games. Journal of Economic Theory, 50(2):381-413, 1990.

[Cooper, 1999] Russell Cooper. Coordination games. Cambridge University Press, 1999.

[Crawford and Sobel, 1982] Vincent P Crawford and Joel Sobel. Strategic information transmission. Econometrica: Journal of the Econometric Society, pages 14311451, 1982.

[Das and Li, 2014] Sanmay Das and Zhuoshu Li. The role of common and private signals in two-sided matching with interviews. In International Conference on Web and Internet Economics, pages 492-497. Springer, 2014.

[Dughmi et al., 2013] Shaddin Dughmi, Nicole Immorlica, and Aaron Roth. Constrained signaling for welfare and revenue maximization. ACM SIGecom Exchanges, 12(1):53-56, 2013.

[Emek et al., 2014] Yuval Emek, Michal Feldman, Iftah Gamzu, Renato PaesLeme, and Moshe Tennenholtz. Signaling schemes for revenue maximization. ACM Transactions on Economics and Computation, 2(2):5, 2014.

[Eső and Szentes, 2007] Péter Eső and Balazs Szentes. Optimal information disclosure in auctions and the handicap auction. The Review of Economic Studies, 74(3):705-731, 2007.

[Ganuza and Penalva, 2010] Juan-José Ganuza and Jose S Penalva. Signal orderings based on dispersion and the supply of private information in auctions. Econometrica, 78(3):1007-1030, 2010.
[Gossner, 2000] Olivier Gossner. Comparison of information structures. Games and Economic Behavior, 30(1):4463, 2000.

[Hajaj and Sarne, 2017] Chen Hajaj and David Sarne. Selective opportunity disclosure at the service of strategic information platforms. Autonomous Agents and Multi-Agent Systems, 31(5):1133-1164, 2017.

[Hajaj et al., 2015] Chen Hajaj, John P Dickerson, Avinatan Hassidim, Tuomas Sandholm, and David Sarne. Strategyproof and efficient kidney exchange using a credit mechanism. In $A A A I-15$, pages 921-928, 2015.

[Jewitt and Li, 2012] Ian Jewitt and D Li. Cheap-talk information disclosure in auctions. University of Oxford, mimeo, 2012.

[Levin and Milgrom, 2010] Jonathan Levin and Paul Milgrom. Online advertising: Heterogeneity and conflation in market design. American Economic Review, 100(2):60307, 2010.

[Morris and Shin, 2000] Stephen Morris and Hyun Song Shin. Rethinking multiple equilibria in macroeconomic modeling. NBER macroeconomics Annual, 15:139-161, 2000.

[Moscarini and Smith, 2001] Giuseppe Moscarini and Lones Smith. The optimal level of experimentation. Econometrica, 69(6):1629-1644, 2001.

[Nahum et al., 2015] Yinon Nahum, David Sarne, Sanmay Das, and Onn Shehory. Two-sided search with experts. Autonomous Agents and Multi-Agent Systems, 29(3):364401, 2015.

[Sarne et al., 2014] David Sarne, Shani Alkoby, and Esther David. On the choice of obtaining and disclosing the common value in auctions. Artificial Intelligence, 215:24-54, 2014.

[Segal-Halevi et al., 2019] Erel Segal-Halevi, Shani Alkoby, Tomer Sharbaf, and David Sarne. Obtaining costly unverifiable valuations from a single agent. In $A A M A S-19$, pages 1216-1224, 2019.

[Shen et al., 2018] Weiran Shen, Pingzhong Tang, and Yulong Zeng. A closed-form characterization of buyer signaling schemes in monopoly pricing. In $A A M A S$ - 18 , pages 1531-1539, 2018.

[Steinert-Threlkeld, 2014] Shane Steinert-Threlkeld. Learning to use function words in signaling games. information dynamics in artificial societies IDAS-14, 2014.

[Wiegmann et al., 2010] Daniel D Wiegmann, Kelly L Weinersmith, and Steven M Seubert. Multi-attribute mate choice decisions and uncertainty in the decision process: a generalized sequential search strategy. Journal of mathematical biology, 60(4):543-572, 2010.

[Wilson et al., 2011] Kurt E Wilson, Roberto Szechtman, and Michael P Atkinson. A sequential perspective on searching for static targets. European Journal of Operational Research, 215(1):218-226, 2011. 